\title{
A Secured Cloud Data Storage with Access Privileges
}

\author{
Naresh Vurukonda, B.Thirumala Rao, B.Tirapathi Reddy \\ Department of Computer Science \& Engineering, KL University, India
}

\begin{tabular}{l} 
Article Info \\
\hline Article history: \\
Received Apr 13, 2016 \\
Revised Jul 18, 2016 \\
Accepted Aug 7, 2016 \\
\hline Keyword: \\
Assured deletion \\
Cloud storage \\
Fine grained \\
Policy based access control \\
Security
\end{tabular}

Article Info

rticle history:

Received Apr 13, 2016

Revised Jul 18, 2016

Keyword:

Assured deletion

Fine grained

Security

\begin{abstract}
In proposed framework client source information reinforcements off-site to outsider distributed storage benefits to decrease information administration costs. In any case, client must get protection ensure for the outsourced information, which is currently safeguarded by outsiders. To accomplish such security objectives, FADE is based upon an arrangement of cryptographic key operations that are self-kept up by a majority of key supervisors that are free of outsider mists. In unmistakable, FADE goes about as an overlay framework that works flawlessly on today's distributed storage administrations. Actualize a proof-of-idea model of FADE on Amazon S3, one of today's distributed storage administrations. My work oversee, esteem included security highlights acclimatize were today's distributed storage administration. our research work proceeds in ensuring the file access control and assured deletion in multi cloud environment and reducing the meta data management, there by the cloud storage become more attractive and many users will adopt the cloud space in order to diminish the data storage cost.
\end{abstract}

Copyright (C) 2016 Institute of Advanced Engineering and Science. All rights reserved.

\section{Corresponding Author:}

Naresh Vurukonda,

Department of Computer Science and Engineering,

KLUniversity,

Vaddeswaram, Guntur, 522502,A.P, India.

Email: naresh.vurukonda@gmail.com

\section{INTRODUCTION}

Cloud storage is a show up administration demonstrates that empowers element and ventures to outsource the warehouse of information reinforcements to remote cloud worker requiring little to no effort. Be that as it may, cloud customers must authorize security confirmation of their outsourced information reinforcements the expanding praise of distributed storage is prevailing associations to analyze moving information out of their own server farms and into the cloud. It is the long-held long for registering as an adequacy [1], can possibly change over an expansive part of the IT company, making programming considerably additionally beguiling as an administration and build, the way IT equipment is planned and get.

Distributed computing alludes to both the applications passed on as administrations over the Internet and the equipment and plan programming in the datacenters that organize those administrations. A methodology framework that addresses the issues of convoluted strategies is characterized and embellished based on the necessities of those approaches, cryptographic enhancements that immeasurably propel authorization capacity of Time-based records, when made, are expressed to have an end time [2].

$\mathrm{ABE}$ viewpoint based encryption is to build up the capacity to decrease cryptographic expenses. At the point when the cloud is made open in pay as you go angle to the well known open we call it as open cloud. Self-satisfied Mug a photograph dissemination Website facilitated terabytes of photographs on Amazon S3 in 2006 and spared a great many dollars on proceed with capacity gadgets utilizing distributed storage for far off reinforcement could discover in the system [3]. Drop box-like machine to move sound/video records from their advanced mobile phones to the unhappiness, given that PDAs regularly have characterized capacity assets. Aside from organization and Government Company, people, third gathering 
worker security make substance to the allotted by the substance worker and authorization of endorsement approaches and client consents.

We started FADE. The first is selective control key utilized by key controller and the second one is information power key utilized by Cloud Client [4]. FADE sums up time-based record ensured cancellation into an all the more fine-grained access called strategy based document settled cancelation, in which documents are join with more pliant document access approach (e.g., time termination, read/compose consents of certify clients) and are totally erased when the consolidate record access strategies are annul and get to be out of date [5]. proceeds in ensuring the file access control and assured deletion in multi cloud environment and reducing the meta data management, there by the cloud storage become more attractive and many users will adopt the cloud space in order to diminish the data storage cost [6].

\section{RELATED WORK ON CLOUD SECURITY AND ACCESS CONTROL}

The cloud computing does not give control over the put away information in cloud server farms. The cloud administration suppliers have brimming with control over the information, they can play out any malevolent undertakings, for example, duplicate, decimating, altering, and so on. The cloud guarantees certain level of control over the virtual machines [7]. Because of this absence of control over the information leads in more prominent security issues than the non specific cloud computing model as appeared in Figure 1. Distributed storage is another business answer for removed reinforcement outsourcing, as it offers a reflection of outright storage room for customers to host information reinforcements in a pay-as you-go way [8]. Time based File guaranteed Deletion is the Existing access [9],[10]. Time-based document settled erasure, which is initially transported in, implies that records can be safely erased and persist for all time remote after a pre-characterized degree. The principle thought is that a record is scrambled with an information key by the proprietor of the document, and this information key is more remote encoded with a control key by a segregated key manager [11],[12]. Sometimes when the data theft by insider is simply passed with the help of creation of decoy file on demand [13].

The key controller is a server that is essential for cryptographic key administration. The control key is time-based, content that it will be totally cleared by the key administrator when a discontinuance time is come to, where the suspension time is portrayed when the record is initially insisted. Without the control key, the information key and thus the information record continue scrambled and are hope to be difficult to reach. In this manner, the fundamental security domain of record guaranteed expunction is that regardless of the fact that a cloud worker does not expel finish up document duplicates from its stockpiling, those documents persist encoded and unrecoverable. Later, the thought of time-based document beyond any doubt cancellation is prototyped in Vanish. Vanish cut an information key into different key shares, which are then accumulated in various hubs of an open Peer-to-Peer Distributed Hash Table (P2P DHT) framework [14]. Individual information put away in the Cloud may contain account numbers, passwords, notes, and other critical data that could be utilized and abused by a rapscallion, a contender, or an official courtroom. This information are reserved, duplicated, and chronicled by Cloud Service Providers (CSPs), regularly without clients' approval and control. Self-Annihilating information for the most part goes for securing the client information's protection. Every one of the information and their duplicates get to be destructed or indiscernible after a client indicated time, with no client mediation. What's more, the decoding key is destructed after the client determined time. To actualize the SADS security framework we are utilizing AES and Random key Generation. Arbitrary Key era is the way toward producing keys for cryptography. A key is utilized to encode and unscramble whatever information is being scrambled/decoded [15].

\section{IMPLEMENTATION}

We name a distributed storage framework brought secure access benefit over cloud information like FADE , which intends to bear the cost of methodology control settled cancellation for record that are available by today's distributed storage administrations. We colleague records with document association strategies that control how documents can be gotten too, we then started arrangement based document settled erasure, in which case are without a doubt cancel and made unrecoverable by anyone when their related record approach strategies are abolish [16]-[18]. We portrays the essential operations. On cryptographic keys in order to accomplish approach control and settled erasure [19]. FADE likewise influences real cryptographic strategies, numbering property based encryption (ABE) and a majority of key controller in view of edge arranged sharing. We execute a model of FADE to show its get up and go, and systematically concentrate on its execution flying when it works with Amazon S3. Our exploratory results give bits of knowledge into the execution security exchange off when FADE is sent by and by. In this paper, we characterize the metadata of Fade being joined to individual information records [20],[21]. We then portray 
how we execute the customer and a majority of key directors and how the customer collaborates with the cloud.

1. Key Controller

2. Cloud user

3. Cloud admin server

4. Policy based access control

5. Policy based assured deletion

\subsection{Key Controller}

Fade is based on a majority of key administrators, each of which is a stand-alone substance that keeps up strategy based keys for access control and guaranteed cancellation. Sorts of keys: Data key, control key, access key, remote client. Numerous arrangements, approach recharging. Arrangement cancellation will be finished by key director.

\subsection{Cloud User}

The one is getting to the approaches set by the cloud chief. Client is legitimate on the off chance that he get to just the arrangements set by the cloud administrator or else he will be distinguishing as a misrepresentation client in the cloud organizing. In the event that the client's arrangements are substantial which doled out for him, then the client can get to every one of the benefits in the cloud organizing.

\subsubsection{Multiple policies}

Arrangements are only the entrance benefits being set by the cloud director on the proprietor's information put away in the cloud server. Active information documents being put away by the proprietor stay on cloud with related arrangement of client characterized record access strategies (e.g., time termination, $\mathrm{read} /$ compose authorizations of approved clients), such that information records are available just to clients who fulfill the document access approaches User keeping in mind the end goal to have entry consent's and for erasure need's sure approaches which are being set by the chief.

\subsection{Cloud Admin Server}

The cloud, kept up by an outsider supplier, gives storage room to facilitating information records for the benefit of various FADE customers in a pay-as-you-go way. Each of the information Documents are connected with a mix of record access policies [3]. FADE is based on the flimsy cloud interface, and accept just the essential cloud operations for transferring and downloading information documents.

\subsubsection{Cloud Manager}

Typically deals with the proprietor's information/documents from the end clients. Part: Manages the entrance consents for an end client who is looking for access to the proprietor documents put away in the cloud server. Cloud chief makes and includes an end client by getting enrolled, wherein he gives the entrance authorizations to access to the proprietor's document put away in the cloud server. Additionally has the ability to close down the clients' framework when he/she tries to get to the documents that has no specific access authorization, where in they will be obstructed as treachery.

\subsubsection{Cloud Server}

Cloud Server gives information storage room to the client/information proprietor to store the information that gives the secured and effective method for putting away the proprietor's information. An asset put away in cloud server has set of access authorizations which are being set by the information proprietor while transferring to the server by means of cloud. Proprietor records put away in cloud server are thusly kept up by the TPA (outsider evaluator). Another critical viewpoint here is that different mists are used to keep their information as secure. In one of the cloud server farm the encoded data put away and another cloud can be utilized to store the keys of the cryptography calculation. With a specific end goal to scramble the data we require solid cryptography calculation such that it may be have keys i.e., public key and/or private key [22]. 


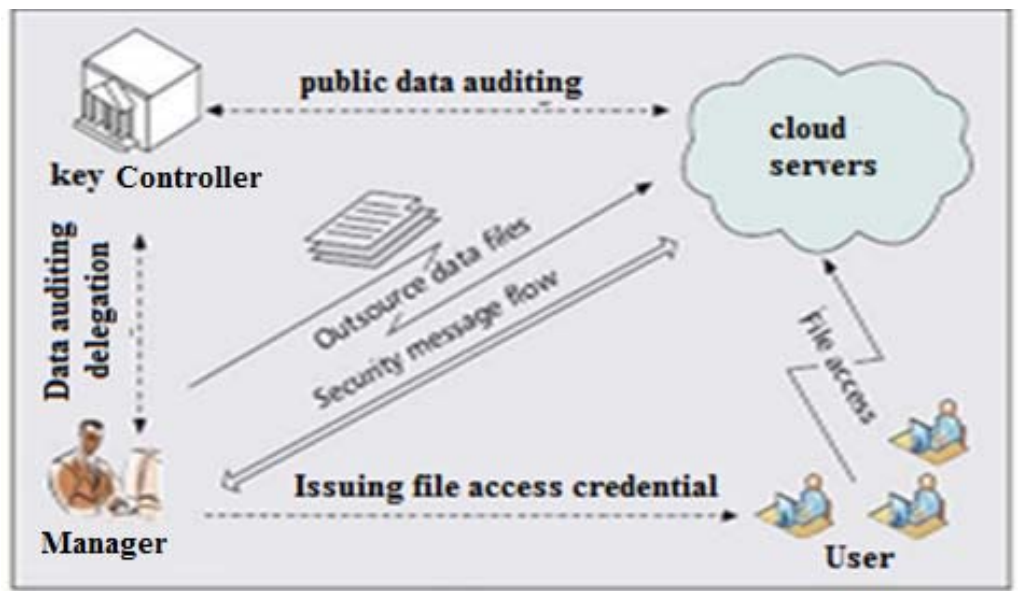

Figure 1. The Block Diagram of cloud storage service

\subsection{Arrangement based access control}

A FADE customer is approved to get to just the records whose related strategies are dynamic and are fulfilled by the customer. It gives emit key to the end client for record transferring and downloading [23].

\subsubsection{Strategies Renewal}

Is the term identified with the entrance authorization's wherein a client solicitations to the cloud supervisor to give the approaches other than which are being distributed to he/her. For the blocked user's (Fraud) keeping in mind the end goal to have admittance to the assets put away in the cloud server need's get to authorization's which are being given by the cloud director when the blocked client goes for asking for the records.

\subsection{Arrangement based guaranteed cancellation:}

A document is erased (or for all time blocked off) if its related approaches are renounced and get to be out of date. That is, regardless of the possibility that a record duplicate that is connected with renounced arrangements, it remains scrambled and we can't recover the relating cryptographic keys to recuperate the document. In this manner, the record duplicate gets to be unrecoverable by anybody (counting the proprietor of the document).

\subsection{Time Performance of Fade}

We first measure the time execution of our FADE Prototype. Keeping in mind the end goal to recognize the time overhead of FADE, we isolate the running time of every estimation into three segments:

- File transmission time, the transferring/downloading time for the information record between the customer and the Cloud.

- Metadata transmission time, the ideal opportunity for transferring/Downloading the metadata, which contains the Policy data and the cryptographic keys related. With the record, between the customer and the Cloud Service Providers.

- Cryptographic operation time, the aggregate time for cryptographic operations, this incorporates the aggregate computational time utilized for performing AES and HMAC on the record, and the ideal opportunity for the customer to organize with the majority of key chiefs on working the cryptographic keys.

- Files are permanently inaccessible based on policies.

\section{RESULTS}

In below, we have shown implementation results succinctly for better understanding in the form of Figure 2, Figure 3, Figure 4 and Figure 5. 


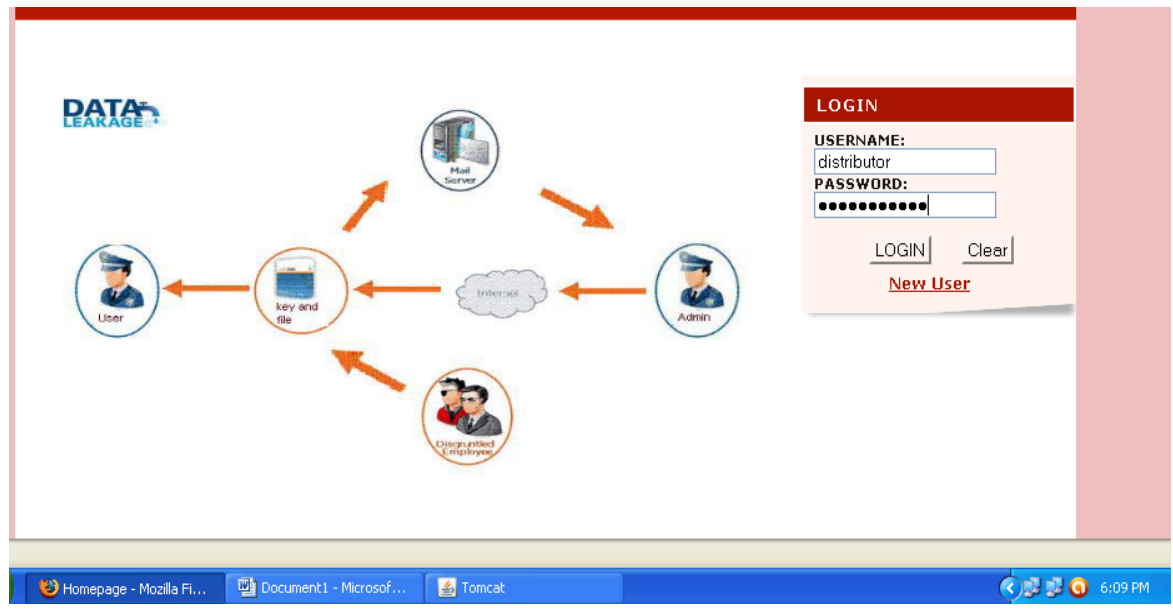

Figure 2. Home page

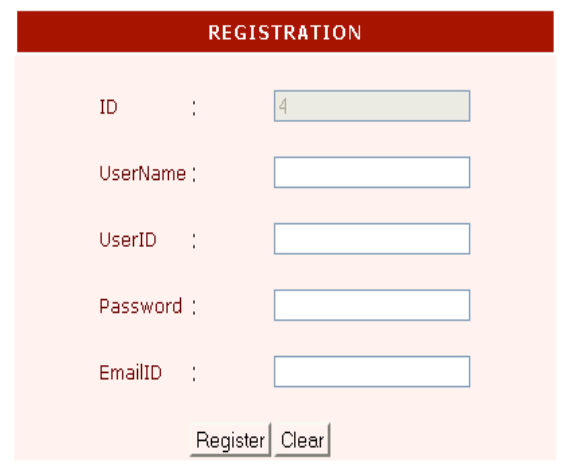

Figure 3. Admin Registration page

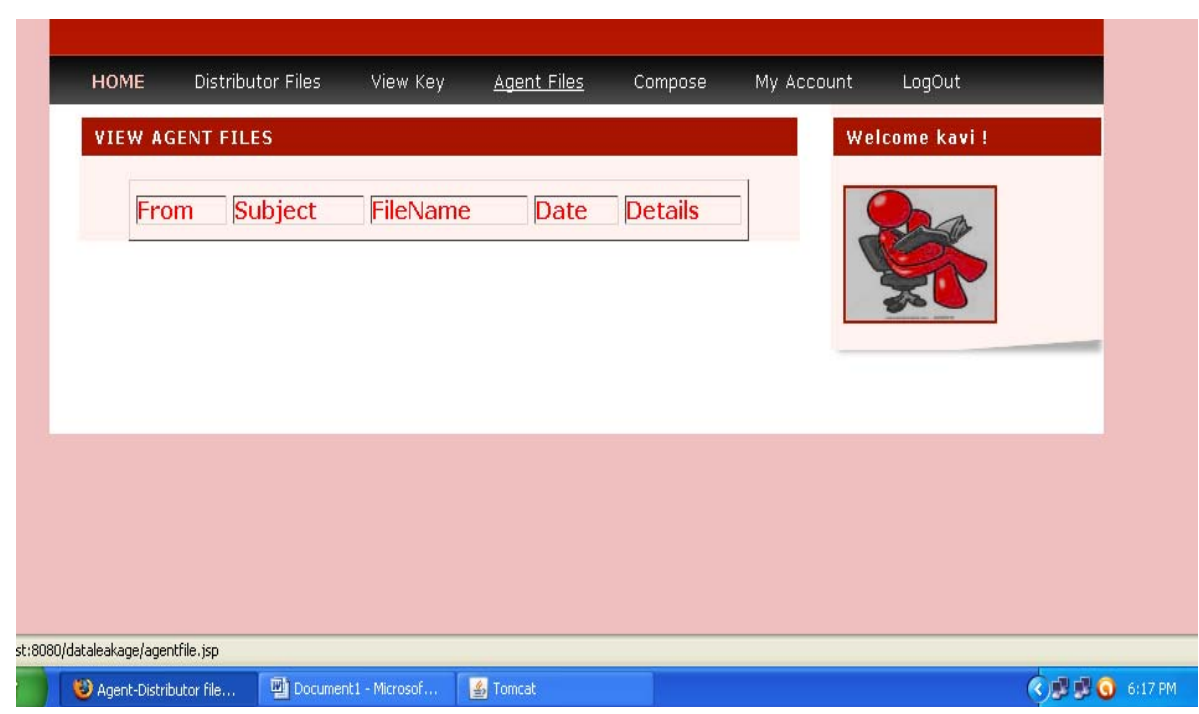

Figure 4. Welcome page 


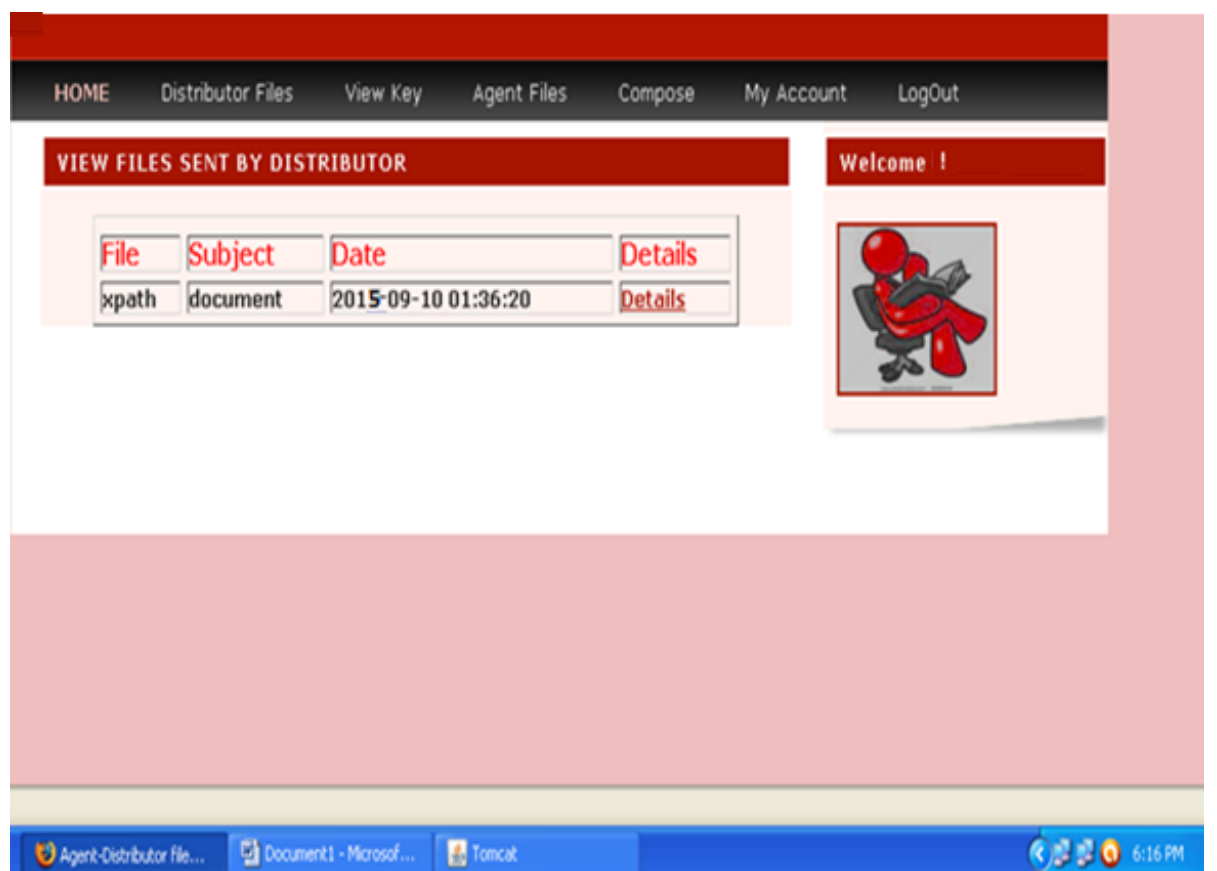

Figure 5. File access page

\section{CONCLUSION}

In This System we proposed a distributed cloud data storage framework brought secure access benefit over cloud information like FADE, It tells about Time based file assured deletion and Vanish data. Which means to give access control guaranteed erasure to documents that are facilitated by todays distributed storage administrations. It partner documents with record access arrangements that control how records can be gotten too. And after that, the present approach based document guaranteed erasure, in which records are definitely erased and made unrecoverable by anybody when their related document access arrangements are denied. Depict the crucial operations on cryptographic keys in order to accomplish access control and guaranteed cancellation. FADE additionally influences existing cryptographic strategies, including the property called attribute based encryption (ABE) and a majority of key supervisors taking into account edge mystery sharing. our research work proceeds in ensuring the file access control and assured deletion in multi cloud environment and reducing the meta data management, there by the cloud storage become more attractive and many users will adopt the cloud space in order to diminish the data storage cost, and also Files which are stored in Cloud are permanently inaccessible after revoking their policies.

\section{REFERENCES}

[1] Goyal V., et al., "Attribute-based encryption for fine-grained access control of encrypted data," in Proc. of the 13th ACM conference on Computer and communications security, Oct 30, ACM, pp. 89-98, 2006.

[2] Bethencourt J., et al., “Ciphertext-policy attribute-based encryption,” Insecurity and Privacy, 2007. SP'07. IEEE Symposium on, May 20, pp. 321-334, 2007.

[3] Tang Y., et al., "FADE: Secure overlay cloud storage with file assured deletion," in Security and Privacy in Communication Networks, Springer Berlin Heidelberg, Sep 7, pp. 380-397, 2010.

[4] Rahumed A., et al., "A secure cloud backup system with assured deletion and version control," in Parallel Processing Workshops (ICPPW), 2011 40th Int. Con. On, Sep 13, pp. 160-167, 2011.

[5] Gunasekhar T., et al., "A Survey on Denial of Service Attacks."

[6] B. T. Reddy, et al., "A survey on assured file deletion in cloud environment."

[7] N. Vurukonda and B. T. Rao, "A Study on Data Storage Security Issues in Cloud Computing," Presented at the Odisha, $2^{\text {nd }}$ Int. ICCC-2016 Conf, Bhubaneswar, Proc. Elseviers-Procedia of Computer Science, Jan 24\&25, 2016.

[8] Mell P. and Grance T., "The NIST definition of cloud computing."

[9] Boldyreva A., et al., "Identity-based encryption with efficient revocation," in Proc. of the 15th ACM conference on Computer and communications security, ACM, Oct 27, pp. 417-426, 2008.

[10] Wang C., et al., "Privacy-preserving public auditing for data storage security in cloud computing," in INFOCOM, 2010 Proceedings IEEE, Mar 14, pp. 1-9, 2010.

[11] Wang W., et al., "Secure and efficient access to outsourced data," in Proc. of the 2009 ACM workshop on Cloud computing security, ACM, Nov 13, pp. 55-66, 2009. 
[12] Yu S., et al., "Attribute based data sharing with attribute revocation," in Proc. of the 5th ACM Symposium on Information, Computer and Communications Security, ACM, Apr 13, pp. 261-270, 2010.

[13] K. Sastry, et al., "Novel Approach for Control Data Theft Attack in Cloud Computing," International Journal of Electrical and Computer Engineering, vol/issue: 5(6), 2015.

[14] Shu X. and Li X., “A Scalable and Robust DHT Protocol for Structured P2P Network.”

[15] M. Sadasivam and R. Dharmaraj, "SADS-Self Annihilating Data Storage system in Cloud Storage Service,” International Journal of Information \& Computation Technology, pp. 0974-2239.

[16] Yun A., et al., "On protecting integrity and confidentiality of cryptographic file system for outsourced storage," in Proc. of the 2009 ACM workshop on Cloud computing security, ACM, Nov 13, pp. 67-76, 2009.

[17] Ruj S., et al., "Decentralized access control with anonymous authentication of data stored in clouds," Parallel and Distributed Systems, IEEE Transactions on., vol/issue: 25(2), pp. 384-94, 2014.

[18] Wan Z., et al., "HASBE: a hierarchical attribute-based solution for flexible and scalable access control in cloud computing," Information Forensics and Security, IEEE Transactions on., vol/issue: 7(2), pp. 743-54, 2012.

[19] Jayalekshmi M. B. and Krishnaveni S. H., "A Study of Data Storage Security Issues in Cloud Computing,” Indian Journal of Science and Technology, vol/issue: 8(24), 2015.

[20] Rani N. A., et al., "A Survey on Data Redundancy Check in a Hybrid Cloud by using Convergent Encryption," Indian Journal of Science and Technology, vol/issue: 9(4), 2016.

[21] Saikeerthana R. and Umamakeswari A., "Secure Data Storage and Data Retrieval in Cloud Storage using Cipher Policy Attribute based Encryption,” Indian Journal of Science and Technology, vol/issue: 8(S9), pp. 318-25, 2015.

[22] T. Gunasekhar, et al., "Mitigation of Insider Attacks through Multi-Cloud," International Journal of Electrical and Computer Engineering, vol/issue: 5(1), pp. 136, 2015.

[23] Yang T., et al., "A Secure Cipher text Self-Destruction Scheme with Attribute-Based Encryption,” Mathematical Problems in Engineering, 2015. 